Hernández-Castilla, R., Murillo, F.J. y Hidalgo, N. (2017). Lecciones Aprendidas del Estudio del Liderazgo Escolar Exitoso. Los casos de España en el Proyecto Internacional ISSPP. Revista de Investigación Educativa, 35(2), 499-518 DOI: http://dx.doi.org/10.6018/rie.35.2.279241

\title{
Lecciones Aprendidas del Estudio del Liderazgo Escolar Exitoso. Los casos de España en el Proyecto Internacional ISSPP
}

\section{Lessons from the International Successful School Principalship Project. The case of Spain in the ISSPP Project}

\author{
Reyes Hernández-Castilla*, F. Javier Murillo** y Nina Hidalgo *** \\ *Universidad Autónoma de Madrid \\ **Universidad Autónoma de Madrid \\ ***Universidad Autónoma de Madrid
}

\begin{abstract}
Resumen
Los estudios sobre el liderazgo escolar es una de las líneas más fructíferas de investigación en las últimas décadas. En concreto, existe una línea de investigación sobre cómo son los líderes de las escuelas exitosas (Day, 2016b, 2017; Day, Gu, E Sammons, 2016; Moos, Johansson, E Day, 2011). El proyecto Internacional ISSPP (International Successful School Principalship Project) se centra en conocer cómo es el liderazgo escolar exitoso en los distintos países que conforman la red. Dentro de este proyecto de envergadura internacional, el presente estudio busca conocer las características tanto personales como profesionales de la dirección escolar exitosa. Para ello, se ha realizado una investigación de enfoque cualitativo a partir de cuatro estudios de casos en la Comunidad Autónoma de Madrid. Para ello se han realizado entrevistas semi-estructuras, grupos de discusión, cuestionarios y observaciones. Los principales resultados señalan que los directores exitosos son aquellos que a) tienen una clara orientación de la escuela, b) muestran un liderazgo humano, c) abren sus escuelas a la comunidad d) adecuan el centro al contexto y e) ejercen un liderazgo pedagógico.

Palabras clave: Liderazgo escolar; Liderazgo exitoso; Directores; Estudio de casos.
\end{abstract}

Correspondencia: Nina Hidalgo. nina.hidalgo@uam.es. C/ Francisco Tomás y Valiente, 3, Campus de Cantoblanco, 28049, Madrid. 


\begin{abstract}
Studies on school leadership are one of the most successful research lines in recent decades. In particular, there is an interesting area of research on how successful school leaders are (Day, 2016b, 2017, Day, Gu, and Sammons, 2016; Moos, Johansson and Day, 2011). The International Successful School Principalship Project (ISSPP) focuses on learning about successful school leadership in the different countries that make up the network. Within this project with an international scope, the present study seeks to know the characteristics both personal and professional of successful school leaders. For this, a qualitative research out of four case studies placed in Madrid (Spain) has been carried out. Semi-structured interviews, discussion groups, questionnaires and observations were also carried out for this purpose. The main results indicate that successful principals are those who a) have a clear direction of the school, $b$ ) show human leadership, c) open their schools to the community d) adapt the school to the context and e) exercise a pedagogical leadership.

Keywords: School Leadership; Successful Leadership; School Principal; Case Studies.
\end{abstract}

\title{
Introducción
}

Este estudio pretende aportar cuatro casos al llamado Strand 1 sobre el liderazgo escolar exitoso del ISSPP (International Successful School Principalship Project). Los directores y directoras observados en el estudio realizado en España son docentes comprometidos, apasionados por el trabajo en el aula y por su escuela. Han sido elegidos por sus compañeros mediante un proceso electoral, pero además cuentan tanto con el reconocimiento de la comunidad educativa como del entorno de la escuela. Estas características los hacen ser singulares. Profundizar en cómo son y cuáles son sus prácticas como directores/as nos ayuda a configurar qué elementos están presentes en el liderazgo escolar exitoso. Este artículo, presenta un estudio realizado por la red de Investigación Internacional sobre Liderazgo Escolar Exitoso en el marco del ISSPP (Day, 2016, 2017; Day, Gu, \& Sammons, 2016; Moos, Johansson, \& Day, 2011) ${ }^{1}$.

El concepto de liderazgo tiene una larga trayectoria, y es una forma de actuar que moviliza e influencia a otros para articular y lograr metas compartidas (Leithwood, \& Riehl, 2005). En cierto modo, el ámbito del liderazgo escolar implica procurar el aprendizaje integral en todos los niveles de la escuela. Autores como Spillane, Diamond, Burch, Hallett, Jita y Zoltners (2002) lo entienden como un proceso de comunicación entre el líder y los docentes. Moos (2009) añade, además, que se requiere una toma de decisiones por parte de los seguidores.

Asimilar el liderazgo escolar con la dirección escolar no es necesariamente riguroso. Sin embargo, en España, la distinción entre éste y la dirección escolar es casi inexistente. En esta misma línea, ya Muñoz-Repiso (1995) habló de dos perfiles diferentes, el profesional y el técnico, como dos aspectos diferenciados. Aunque quedarnos en esta distinción no es suficiente. En muchas investigaciones, como las realizadas por

1 Estudio ha sido financiado por el Ministerio de Economía y Competitividad bajo el Plan Estatal de Investigación Científico y Técnica cuyo título es: "Escuelas en contextos socio-económicamente desafiantes: una aproximación desde la educación para la justicia social" (Referencia: EDU2014-56118-P) I.P: F. Javier Murillo y Reyes Hernández-Castilla. 
Hopkins, Ainscow y West (1994), Stoll y Fink (1998) o Fullan (2003) van más allá, y los convierten no tan solo en líderes de las escuelas en términos jerárquicos sino también en impulsores del cambio hacia la mejora de la institución escolar.

El proyecto del ISSPP que enmarca este estudio bebe de tres fuentes fundamentales (figura 1). En primer lugar la eficacia Escolar, que tradicionalmente ha estudiado el rendimiento de los estudiantes y el liderazgo escolar. En segundo lugar, la investigación sobre el cambio y la sostenibilidad en la mejora de los centros (Hargreaves, \& Fink, 2003, 2008). La tercera fuente corresponde a las contribuciones sobre cuáles son esas prácticas del liderazgo escolar exitoso como por ejemplo en los estudios de Leithwood y Riehl (2005), Leithwood y Jantzi (2006) y Day, Sammons, Leithwood, Hopkins, Harris, Gu, \& Brown (2010). Los hallazgos de las investigaciones llevadas a cabo en el marco del ISSPP sobre los lideres escolares exitosos, los describen como aquellas personas que establecen metas comunes, desarrollan a las personas y gestionan satisfactoriamente su relación con la comunidad (Day et al., 2009, 2010; Drysdale, \& Gurr, 2011, 2016).

El estudio Internacional sobre Dirección Escolar Exitosa, International Succesful School Principaship Project (ISSPP), tuvo su inicio en 2001, en Inglaterra y Estados Unidos; aunque pronto se sumaron los países Escandinavos (Noruega, Suecia y Dinamarca), China (Shangahi y Hong Kong) y Australia (Victoria y Tasmania). Dichos estudios sucesivos han aportado numerosa producción: a) el liderazgo escolar exitoso (Gurr, Drysdale, \& Mulford, 2006; Gurr, \& Drysdale, 2003; Swann, Gurr, Ford, Drysdale, Hardy, \& Di Natale, 2003), b) el liderazgo para los agentes sociales de la comunidad; y c) el liderazgo para el aprendizaje y mejora de los resultados de los estudiantes (Mulford, Silins, \& Leithwood; Seashore, Anderson y Wahlstrom, 2004). Además, se ha buscado comprender y conocer las concepciones de la cultura y la política (Foster, 1986) así como la perspectiva del liderazgo escolar distribuido (Chi-Kin, \& Day 2016a; Spillane, Camburn, \& Stitziel, 2007).

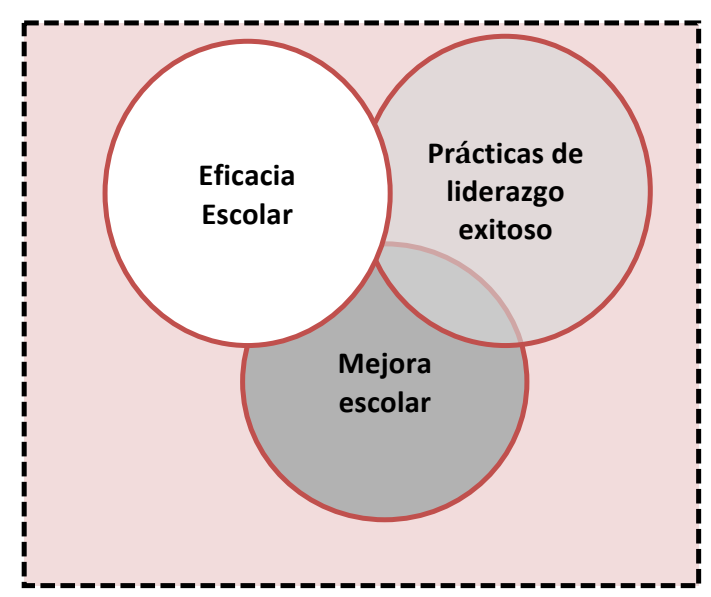

Figura 1. Fuentes para el International Succesful School Principalship Project.

Elaboración propia. 
Estos estudios que ayudaron a configurar la red del ISSPP hallaron cinco elementos comunes que definen un liderazgo exitoso (figura 2): a) un liderazgo para el aprendizaje, b) la importancia de la formación de los directores, c) el cuidado de los estudiantes, d) su focalización hacia el éxito (entendido en un amplio sentido) y e) la rendición de cuentas (Drysdale, \& Gurr, 2016; Møller, 2016; Wang, Gurr, \& Drysdale, 2016).

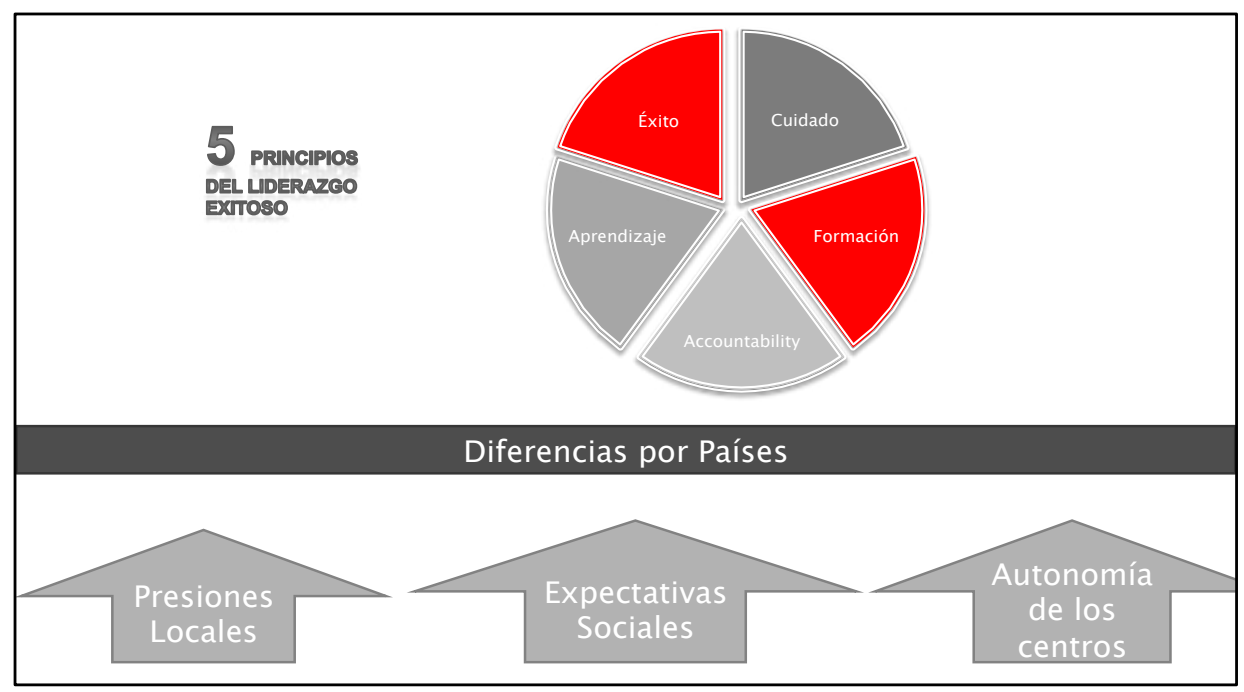

Figura 2. Principios que configuran un liderazgo exitoso para el International Succesful School Principaship Project. Elaboración propia.

Las diferencias observadas en la comparación internacional de casos vienen dadas por tres elementos: las presiones locales, las expectativas sociales y la autonomía de los centros.

La segunda pregunta de investigación enmarca la descripción de éstos líderes y qué les caracteriza: a) un compromiso apasionado y sostenido junto con una rendición de cuentas personal; b) su capacidad para gestionar los dilemas y las tensiones con un criterio ético; c) se focalizan en el aprendizaje de los estudiantes; y d) hacen un gasto emocional y racional para lograr el buen funcionamiento del centro (Day, \& Leithwood, 2007; Harris, Leithwood, Day, Sammons, \& Hopkins, 2007). En el estudio comparado de los resultados obtenidos en Australia, Dinamarca, Noruega, Suiza, Inglaterra y Estados Unidos, los autores hallaron que son líderes con una mentalidad abierta, con capacidad para la toma de decisiones, con una escucha activa, con optimismo, con un gran sentido del respeto y el cuidado, y con altas expectativas hacia sus profesores (Day, \& Hong, 2016). De la conclusión de este estudio comparado previo podemos aprender que los líderes exitosos son personas comprometidas con su trabajo y profundamente interesados en los otros (tanto estudiantes como docentes).

Al revisar la literatura sobre los resultados obtenidos en los años recientes (por ejemplo, Leithwood, \& Jantzi, 2006; Leithwood, \& Riehl, 2005; Leithwood, Seashore, 
Anderson, \& Wahlstrom, 2004; Robinson, 2007) hemos podido observar elementos comunes que caracterizan el liderazgo exitoso:

a) Los líderes establecen la dirección o la misión del centro de manera que su liderazgo crea y da sentido e intención a la organización, desarrollando una visión compartida del futuro. Asimismo, ayudan a construir un consenso sobre lo que es prioritario a corto plazo y muestran unas altas expectativas respecto al trabajo de sus colegas (Leithwood, 2005).

b) Comprenden y desarrollan a las personas. Esta es una de sus principales tareas: construir un entorno en el que las relaciones con los estudiantes sean óptimas, lo que requiere que la estructura y la cultura de la escuela apoye su capacidad como docentes y como personas (Day, \& Hong, 2016).

c) Construyen la escuela como una comunidad. Es una organización que debe ser sostenida por las estructuras organizativas, pero no es suficiente para que éstas sean eficaces y exitosas, deben ser comunidades, entendiendo que han compartir una identidad y unas normas.

d) Gestionan la docencia y el currículum. Si el director/a centra su atención, y consecuentemente los docentes, en determinadas metas, niveles y criterios de la programación esto supone que con mayor probabilidad los estudiantes lograrán las competencias deseadas.

e) Lideran el entorno. Las escuelas son profundamente dependientes de su entorno político, administrativo, comunitario, profesional, cultural, etc. Por ello, una de las tareas principales de los directores y directoras es gestionar y liderar las relaciones con el entorno. Son capaces de comprender e interpretar las expectativas de los padres y madres, y lograr que la escuela sea vista como exitosa, bien a través de las diferentes referencias objetivas de evaluaciones externas, o bien con los informes de la inspección o los políticos locales que influyen en ellas (Weick, 2001).

\section{Método}

\section{Objetivos del estudio}

Este estudio ha sido realizado en el contexto del Proyecto Internacional sobre Liderazgo Escolar Exitoso (ISSPP) cuyo objetivo principal es describir las características personales y profesionales de la dirección escolar exitosa. Como concreción de este objetivo, se han caracterizado sus prácticas así como sus estrategias de gestión y liderazgo.

\section{Enfoque metodológico}

La metodología utilizada en esta investigación es un estudio de casos desde una multiperspectiva, cuyo diseño e instrumentos han sido validados y empleados para las comparaciones internacionales. Los cuatro casos aquí presentados forman parte del Proyecto Internacional sobre Liderazgo Escolar exitoso (ISSPP) iniciado en 2001, que cuenta con tres subestudios: cualitativo, cuantitativo y longitudinal. 


\section{Participantes e instrumentos de recogida de información}

El proceso de selección de los cuatro directores/as participantes ha utilizado diferentes criterios sumativos: a) una evaluación positiva del trabajo realizado por el director/a; b) han de ser reconocidos por sus pares profesionales como líderes exitosos; c) a sus centros asiste un alumnado heterogéneo; d) obtienen resultados satisfactorios en las pruebas externas de manera sostenida en el tiempo y con la presencia del director/a estudiado. Los centros elegidos con estos criterios están situados en la zona Centro, zona Norte y zona Sur de Madrid.

Tabla 1

Centros participantes

\begin{tabular}{lcccc}
\hline & Centro 1 & Centro 2 & Centro 3 & Centro 4 \\
\hline Titularidad & Pública & Pública & Pública & Pública \\
\hline Ubicación & Urbana & Urbana & Urbana & Urbana \\
Nivel de Enseñanza & CEIP & CEIP & IES & IES \\
№. Estudiantes & 440 & 417 & 750 & 1200 \\
$\begin{array}{l}\text { №. Docentes } \\
\text { №. De Personal No } \\
\text { docente }\end{array}$ & 10 & 33 & 65 & 80 \\
\hline
\end{tabular}

Los instrumentos utilizados, validados internacionalmente, han sido entrevistas focales estructuradas, grupos focales, y cuestionarios. Además se han realizado observaciones de los centros con un protocolo consensuado internacionalmente. En la tabla 1 se muestra el número final de informantes de cada uno de los centros educativos participantes en la investigación.

Tabla 2

Entrevistas y grupo de discusión

\begin{tabular}{lcccc}
\hline & Centro 1 & Centro 2 & Centro 3 & Centro 4 \\
\hline Director/a & 3 & 3 & 3 & 3 \\
Jefe/a de Estudios & 1 & 1 & 1 & 1 \\
Secretario/a & 1 & 1 & 1 & 1 \\
Coordinador de Ciclo/ & 2 & 2 & 2 & 2 \\
Jefe de Departamento & 3 & 3 & 3 & 3 \\
Profesores & 3 & 3 & 3 & 3 \\
Madres/padres & $11(4 \mathrm{~h} \mathrm{y} 7 \mathrm{~m})$ & $9(3 \mathrm{~h} \mathrm{y} 6 \mathrm{~m})$ & $10(3 \mathrm{~h} \mathrm{y} 7 \mathrm{~m})$ & $8(1 \mathrm{~h} \mathrm{y} 7 \mathrm{~m})$ \\
\hline Grupo de discusión
\end{tabular}

${ }^{*} \mathrm{~h}=$ hombres y $\mathrm{m}=$ mujeres 
Tanto las entrevistas focales como los grupos focales están organizados en cinco elementos: a) Principales desafíos del centro; b) Estrategias utilizadas con éxito para afrontar los desafíos; c) Cualidades percibidas del director y contribución del equipo de gestión; d) Integración de alumnos procedentes de diferentes entornos sociales y culturales; e) Problemas persistentes y nuevos retos (tabla 2).

En las entrevistas se le ha preguntado sobre la historia de la escuela, su situación actual y los retos que deberá abordar. Se les ha pedido una descripción de la estructura de gestión del centro, de su estructura curricular, así como las actividades extracurriculares que se llevan a cabo. Asimismo, se les ha solicitado que identifiquen los factores clave de su éxito. Las entrevistas han ido orientadas también a conocer la implicación de las familias en la escuela así como aspectos motivacionales y de satisfacción de los docentes. Tanto al director/a como a las familias y los docentes se les ha pedido que describan cómo ejercen el liderazgo. Quizás una de las preguntas fundamentales ha sido la descripción de retos del centro para poder analizar cuáles son sus focos de atención y su mirada al futuro.

\section{Análisis de los datos}

Para profundizar en las entrevistas y los grupos focales se ha utilizado como unidad de análisis cada caso/escuela. Una vez que estos casos se analizan individualmente se ha procedido a trabajar las categorías propuestas por el estudio internacional del ISSPP a través de elementos de comparación y de constatación de evidencias que ratifiquen la presencia o ausencia de estos hallazgos.

Los datos han sido estructurados y categorizados en base a su contenido y a su sentido semántico (Strauss, \& Corbin, 2002). El proceso seguido para el análisis de los datos ha seguido los siguientes pasos con apoyo del software Atlas.ti 7:

1. Generación de la Unidad Hermenéutica a la que asignamos los documentos primarios y generamos las familias de éstos organizadas en función de los casos (escuelas).

2. Reducción de los datos: Análisis de frecuencias de los términos. Selección de citas con los fragmentos de las entrevistas y focus group que mejor responden a nuestra pregunta de investigación.

3. Análisis mediante la codificación de cada caso individualmente utilizando las categorías a priori propuestas por el estudio internacional del ISSPP y las emergentes de los datos primarios. Posteriormente, se ha procedido a la elaboración de supercódigos utilizando operadores booleanos y al re-análisis de los datos a través de los supercódigos.

4. Construcción del modelo a través de la generación de redes semánticas que vinculan códigos, supercódigos y grandes categorías.

5. Extracción de conclusiones. 
Tabla 3

Fases del proceso de análisis del contenido

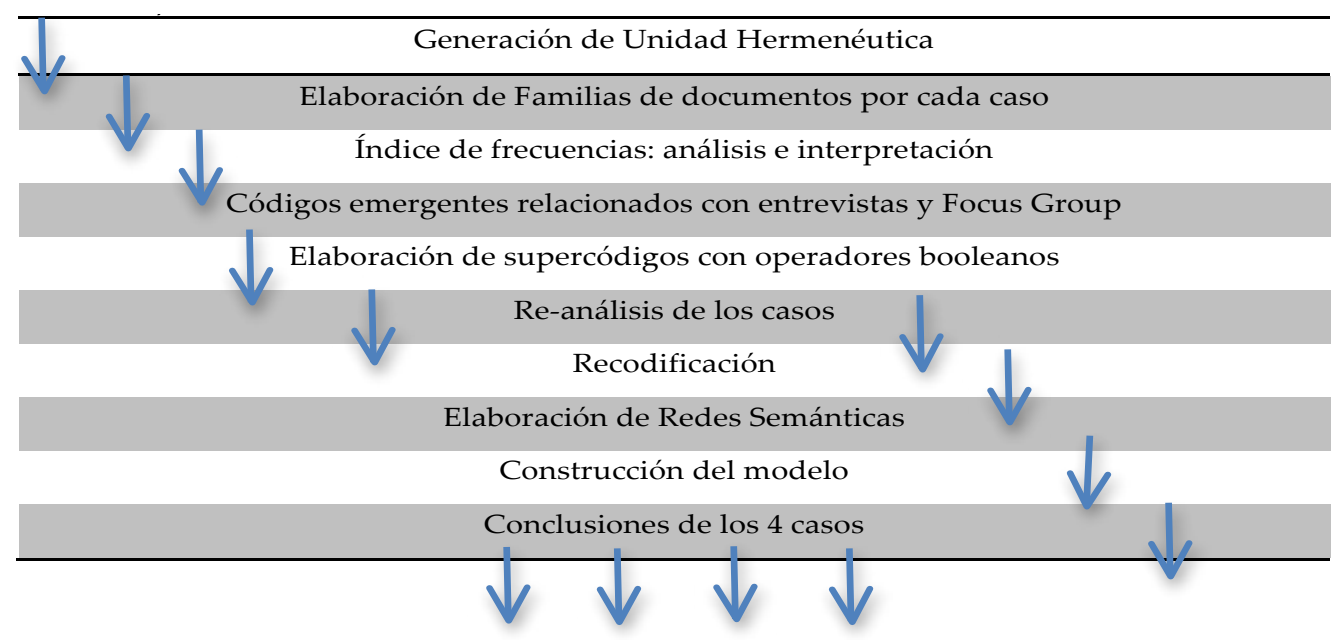

Los tópicos de estos instrumentos los presentamos en un mapa conceptual con las diferentes preguntas de investigación y categorías iniciales de análisis presentes en el estudio internacional (figura 3). En él aparece un gran elemento contextual en el que se inscriben: a) la situación de centro, b) su contexto; y c) la relación con el entorno. Esta primera gran categoría también está incluida las familias. Y, como antecedente a la situación actual de la escuela, emergen dos elementos más: la historia del centro y del propio director/a. Un segundo elemento incluye las relaciones entre los miembros de la comunidad educativa en la que están asignadas: a) las relaciones con los estudiantes, b) sus familias; y c) la participación e implicación en la escuela. Un tercer elemento, está configurado por aspectos directamente vinculados al éxito de los estudiantes y al ejercicio del liderazgo exitoso. Y, por último: se analizan otros elementos y categorías conectadas con las prácticas de liderazgo exitosas: a) el desarrollo de los docentes, b) la gestión del currículo, o c) la gestión del centro. Todas ellas se manifiestan relevantes en la revisión de la literatura sobre liderazgo exitoso.

Tabla 4

Categorías de análisis a priori

\section{Estructura de las entrevistas}

$\checkmark \quad$ Principales desafíos del centro

$\checkmark \quad$ Estrategias para afrontar los desafíos

$\checkmark$ Cualidades percibidas del director y contribución del equipo de gestión

$\checkmark \quad$ Integración de los alumnos de otros entornos sociales y culturales

$\checkmark \quad$ Problemas persistentes y nuevos retos 


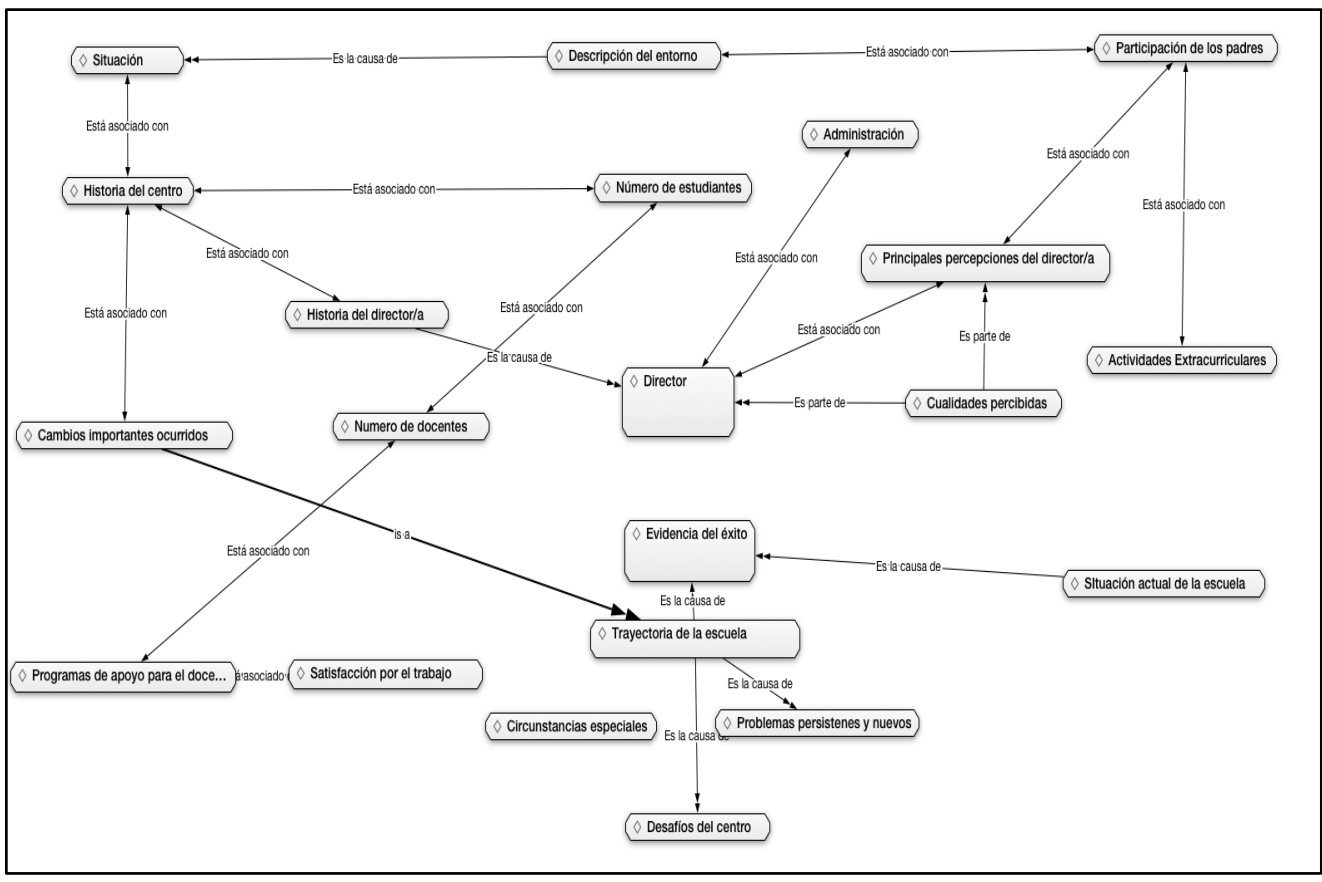

Figura 3. Categorías iniciales de análisis del estudio

\section{Resultados}

Con el propósito de conocer las características y prácticas del liderazgo escolar exitoso se han organizado los resultados en dos apartados. En el primero, presentamos cada uno de los líderes estudiados con la finalidad de conocer su realidad y particularidades. En segundo lugar, pasamos a detallar las prácticas de liderazgo exitoso de los participantes halladas en la presente investigación.

\section{Cuatro docentes con liderazgos escolares exitosos}

En esta investigación en España se ha estudiado en profundidad dos centros de Educación Primaria y dos de Secundaria. Los líderes seleccionados son tres mujeres y un hombre, todos ellos con poco más de cincuenta años. A continuación presentamos la realidad y peculiaridades de cada uno de ellos. Veámoslas.

\section{a) Pilar, directora de un Colegio de Educación Infantil y Primaria}

Es una docente experimentada, treinta años, con un gran interés por capacitarse pedagógicamente. Ha desempeñado diferentes tareas de responsabilidad en el centro. Fue miembro del equipo directivo. Durante doce años ha sido Jefa de Estudios. Ejercer la dirección le gusta. Es calmada y eficaz. Manifiesta un compromiso ético con la educación y la sociedad. 
b) Blanca, de mediana edad, ejerce la dirección también en un Centro de Educación Infantil y Primaria

Blanca es una docente de larga trayectoria, destaca ante sus compañeros por su compromiso y por tener claros los objetivos. Es capaz de trabajar en equipo, y promueve proyectos innovadores. Consiguió cambiar la percepción que tenían de ella y conseguir ser su líder.

c) Diego, un director en Educación Secundaria con una trayectoria de docente en otros centros educativos, tanto públicos como privados

Formó parte del equipo directivo precedente y eso hizo que asumir la dirección fuera un paso natural. Lleva en la dirección 16 años, por lo que podemos describirle como un director experimentado que sigue disfrutando de su docencia y de los estudiantes. Para la toma de decisiones busca soluciones con su equipo y se plantea las carencias como un reto, y toma decisiones de manera compartida. Ha logrado el apoyo y el respeto del claustro.

\section{d) Esperanza, una directora de un Instituto de Educación Secundaria}

Quizá la característica más destacable de Esperanza es su carácter conciliador, que aúna voluntades. Es docente en la escuela desde su creación. Siempre ha estado implicada y asumiendo diferentes responsabilidades en ella. Descubrió que ser docente es lo que más le gusta y se siente comprometida con la escuela pública. En voz de su equipo de dirección, tiene la capacidad para dirigir el centro y continuar con las prácticas exitosas considerando las dificultades como nuevos retos.

\section{Resultados globales}

En primer lugar si observamos el índice de frecuencia en valores absolutos en cada uno de los cuatro centros se muestra la importancia de determinados elementos en la dirección escolar exitosa. En este sentido, aparece el centro como término más frecuente. Es decir, la visión del conjunto, con todos los elementos que implican. En segundo lugar, un elemento también muy interesante: los estudiantes. El foco de su trabajo no es la gestión sino los alumnos y alumnas del centro. Así mismo, un elemento significativo es que aparece él o ella, lo que interpretamos como una elevada identidad con su papel de dirección. Así mismo está el entorno, los padres y madres del centro educativo, y finalmente el equipo. Por tanto, son los aspectos personales los que están presentes de manera prioritaria.

Un segundo grupo de términos hacen referencia a los resultados (recordemos que hablamos de un liderazgo escolar exitoso, que obtiene buenos resultados en pruebas externas), las actividades, el trabajo, la educación como mirada pedagógica y el proyecto. Esos son los términos clave en un liderazgo escolar exitoso. 


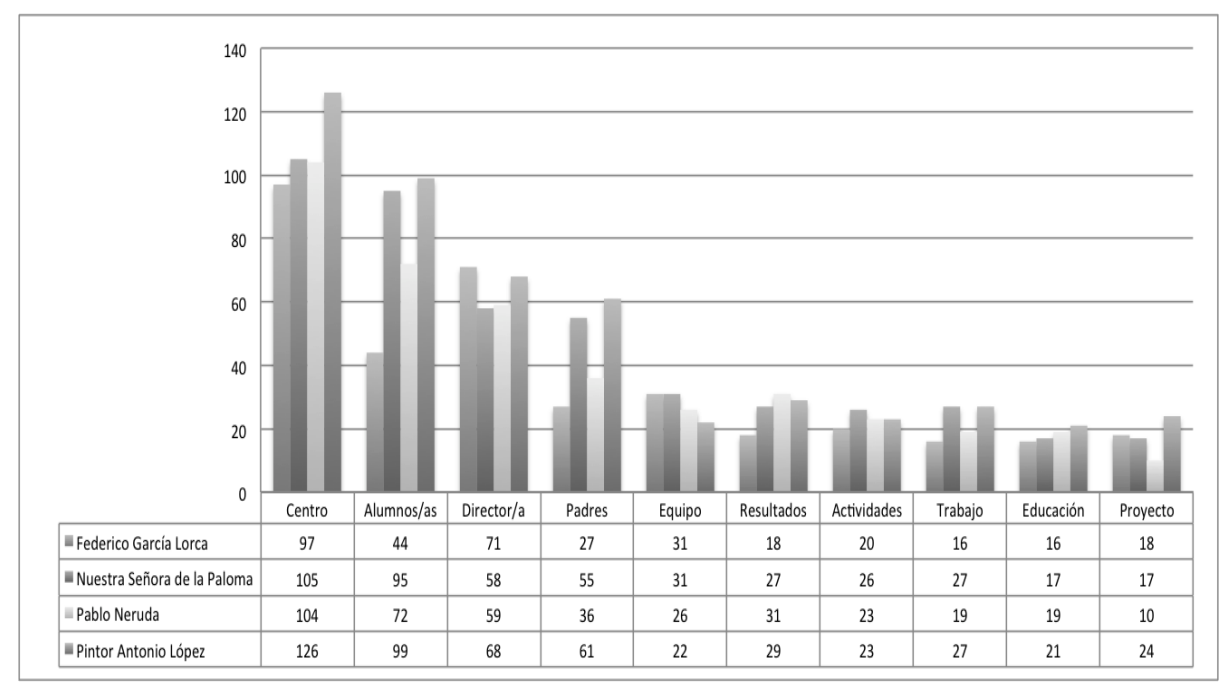

Figura 4. Índice de Frecuencia por Centro Educativo

Para tener una visión de conjunto y qué porcentaje implica cada uno de estos términos, mostramos un gráfico de sectores donde el 27\% de los términos más frecuentes es el "centro", un 19\% los "alumnos/alumnas," el 16\% el "director/a", 7\% "los resultados" y "el equipo", un 6\% "las actividades" y "el trabajo", un 5\% la "educación", y un 3\% "los padres". Estos porcentajes se han obtenido a partir de la suma de la frecuencia en cada centro de estos términos, los cuales han sido seleccionados como los términos más frecuentes en el discurso de docentes y directores entrevistados.

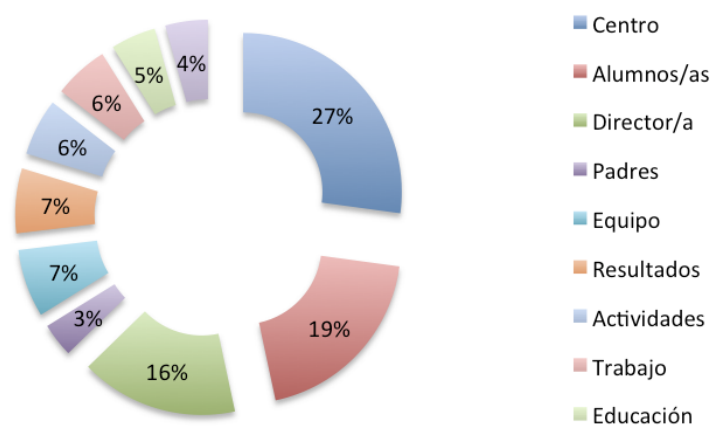

Figura 5. Porcentaje de frecuencia global

\section{Prácticas de liderazgo escolar exitosas}

Del estudio en profundidad de los cuatro directores y sus escuelas se obtienen algunos elementos comunes que son interesantes. Para presentar los principales hallazgos 
hemos utilizado para el análisis las categorías planteadas que Moos (2009) y Leithwood y Riehl (2005) dentro del marco del ISSPP. Las categorías empleadas para el análisis de los líderes escolares exitosos se agrupan entorno a estos elementos: a) establecen la dirección del centro, b) comprenden y desarrollan a las personas; c) diseñan y gestionan las comunidades; d) gestionan el currículum y la docencia; e) lideran el entorno.

\section{a) Establecen la dirección de la escuela}

Los directores/as exitosos ejercen su liderazgo, y con ello, consiguen una visión compartida del futuro de la escuela ayudando a crear un consenso sobre las metas más adecuadas, mostrando además unas altas expectativas hacia sus compañeros/as. La percepción del equipo directivo es que trabajan con empeño y van cumpliendo uno a uno los objetivos propuestos. En este sentido, asumen el liderazgo del centro acorde con un plan de trabajo establecido en el Proyecto de Dirección. Aportan una trasparencia en la información que permite el diálogo y la evaluación permanente de los procesos establecidos. Es decir, tiene una visión de conjunto del centro desde lo más cotidiano y básico a una focalización hacia el aprendizaje de los estudiantes, con una visión optimista de este aprendizaje lo que manifiesta unas altas expectativas en las posibilidades de sus estudiantes.

...no solamente convivencia, sino que el espacio sea agradable: la luz, la limpieza; pues para mí el centro lo concibo como un proyecto, es decir, como profesora me he implicado mucho porque si yo estoy a gusto, yo enseñaré a gusto y si mis alumnos aprenden bien, aprenderán mejor. La responsabilidad que yo tomo es que de alguna manera me toca empujar ese proyecto (...). [D1, SEC, p.5]

Estos directores se caracterizan por tener una visión muy precisa sobre qué se pretende, lo cual ayuda al cumplimiento de los objetivos, a compartirlos y a evaluarlos. La planificación de las prioridades es explícita y conocida. Se basa en el conocimiento de las necesidades y prioridades de escuela:

La organización es una de los elementos básicos. Nosotros decimos que ponemos tres ejes en este centro cuando explicamos a los padres: uno es la convivencia en el centro, es esencial crear una buena convivencia y respeto mutuo para trabajar, otra es lo que haríamos todos los proyectos es un centro (....). El otro punto es la acción tutorial, que para nosotros es importantísima. [D2,PRI, p.2]

b) Comprensión y preocupación por el desarrollo de las personas

Teniendo en cuenta que los docentes son las personas más relevantes en el aprendizaje de los estudiantes, se necesita proporcionar la posibilidad de que construyan las condiciones óptimas de enseñanza. Ello implica que la estructura y la cultura del centro favorecida por el director/a apoye la formación y el desarrollo personal docente: 
La primera cuestión es que intentamos ser equipo, (...) y conciliar vida familiar y profesional, de manera que se trabaja muy duro, pero intentamos no trabajar en el centro más de los necesario, pero no por trabajar más horas es más efectivo el trabajo. [D4, PRI, p.4]

Además de esta racionalización de las tareas para ser efectivos en el trabajo hay una real preocupación por la persona. En este sentido, se busca la cercanía, ser uno mas. Cuidan los afectos y lo combinan con una libertad para tomar decisiones. Esto lo logran siendo accesibles. Su idea es que el:

"liderazgo tiene que estar al pié del cañón perenemente. El director es uno más del centro, forma parte del núcleo sólido. El es muy trabajador, no escatima esfuerzos ni tiempo... en general escucha". [P1, SEC, p.1]

\section{c) Diseñando y gestionando comunidades}

Las escuelas son organizaciones por definición, pero si queremos que éstas sean eficaces y exitosas además deben ser comunidades (Bolívar, 2000). Han de compartir un sentido de identidad así como unas normas y metas comunes (Crow, Day y Møller, 2016). En el ejercicio del liderazgo se ayuda a que todos los miembros de la comunidad estén suficientemente comprometidos con la misión y el proyecto de esta institución.

Por ello, en los casos observados, los directores/as viven el centro como una comunidad, como un lugar de interacción de grupos. El buen clima, hace que se puedan potenciar las relaciones de manera que los tiempos dedicados al trabajo sean satisfactorios. Se manifiesta en una satisfacción por el trabajo:

Me encanta mi profesión entonces entiendo que si yo colaboro para que el centro vaya bien, me voy a sentir bien. Veo el centro como una comunidad de personas en el que cada uno tiene su vida pero que tenemos que funcionar de una manera comunitaria. [D3, SEC, p.1]

Esta comunidad está compuesta por docentes en distintas circunstancias laborales, permanentes, interinos, con mayor y menor formación y experiencia (además de los estudiantes, familias, y personal no docente). Pero todos forman parte de la comunidad, son acogidos e integrados en el grupo. Se cuidan las relaciones, buscando la convivencia y construir comunidad:

La mayoría [la gente] quiere estar. Tenemos muchos interinos, lo que intentamos es que se impliquen porque es importantísimo, pues hay interinos que se van encantados, además les agradecemos el trabajo. Hacemos cosas que mantengan un poco la convivencia entre nosotros, después cuando regresan podrían, entonces, pedir cualquier otro centro de la localidad, vuelven a pedirnos el centro, entonces puede decirse que se encuentran a gusto. [D4,PRI, p.5] 


\section{d) Gestión del currículum y la metodología docente}

Si la dirección de la escuela se focaliza en unos objetivos y criterios de aprendizaje, ello hace que la programación se revalorice, y por tanto, exista una mayor posibilidad de que los estudiantes adquieran estas competencias deseadas. El hecho mismo de que sea algo prioritario para la gestión del centro facilita que los recursos, el tiempo dedicado, etc., permitan el logro de los objetivos. En palabras de uno de los directores:

Quien lleva la parte pedagógica son los Jefes de Departamento, y yo los coordino semanalmente a través de la Jefe de estudios. Ellos tienen que presentar sus programaciones didácticas. Yo tengo que revisarlo y tengo en cuenta que todo se adapta a la normativa, e intentar impulsar y convencerles de que es bueno desarrollar estrategias metodológicas y hacerlo. Es decir, mi parte es que se respete la norma, cuidar de que los alumnos sean evaluados equitativamente, (...) es impulsar nuevas metodologías, nuevos proyectos. Esa es la parte que yo puedo hacer. [D4,PRI, p.3]

En este sentido, la dirección del centro cuando manifiesta con claridad la importancia de los objetivos, cuáles son y su clara apuesta por el logro de éstos logra que sea una meta conocida y compartida. Para conseguirlo realiza acciones constantes de promoción, coordinación y revisión de las metodologías. También la evaluación tiene un papel crítico, por que es el reflejo de lo que se espera que los alumnos sepan y adquieran en el proceso de aprendizaje. Sirve además como un elemento de monitorización de las programaciones y además permite reflexionar sobre las metodologías didácticas utilizadas en la medida en que son eficaces para el logro de los objetivos.

La innovación supone concebir las carencias o demandas como retos. Los directores/as impulsan y participan coordinando proyectos éxitos relacionados con las Tecnologías de la Información y la Comunicación, pero también, por ejemplo, en programas relacionados con las Ciencias Experimentales, con la Convivencia o el Bilingüismo. Son proyectos valorados y premiados por sus iguales.

\section{e) Liderando en el entorno}

Las escuelas son organizaciones sociales que dependen del entorno político, administrativo, cultural, económico y medioambiental en donde se encuentran. Por esta razón los directores/as como representantes de la comunidad educativa han de ser capaces de interpretar las expectativas y las demandas del entorno. Es fundamental liderar estas relaciones y apoyarse en ellas para el logro de una interacción adecuada con éste (Weick, 2001).

Esta apertura a la comunidad implica muchos tipos de relación. Por un lado, los alumnos/as asisten a otros centros, participando en experiencias de intercambio entre los centros. Además se relacionan con la Administración Educativa de la que dependen, a la que rinden cuentas, tanto en la organización de sus recursos humanos, apoyos la docencia, etc. Las diferentes administraciones son un apoyo para los centros, pero también buscan vínculos con la sociedad por ejemplo: mediante organizaciones no gubernamentales, asociaciones etc. Esta apertura se concreta también 
en relaciones internacionales mediante el intercambio con otras escuelas extranjeras, estableciendo vínculos con otros colegas, dentro y fuera de la localidad:

El papel mío como directora son las relaciones externas, las que tengo que cuidar: con la Administración educativa, con la Consejería, al Ayuntamiento, que nos ofrece muchas ayudas... por ejemplo: un desbroce de parcela gratuito que tenemos, nos cede unas pistas deportivas para el Ciclo Formativo de Grado Superior, utilizamos las instalaciones. Tenemos Asociación de jóvenes, Asociación de la Talla en Madera, la Universidad Popular de aquí... Yo ayudo a los profesores que están en todos los proyectos, mantengo, como directora, las relaciones internacionales con los directores de las escuelas europeas, entonces asisto a seminarios, invito a jornadas aqui, entonces mi trabajo es relaciones externas. [D1, PRI, p.3]

\section{Discusión y conclusiones}

El liderazgo escolar sin duda es una de los elementos claves en las políticas educativas internacionales debido a su papel decisivo en la mejora de los resultados escolares por la potencialidad que tiene para favorecer capacidades tanto de los docentes como del entorno de la escuela. Uno de los debates actuales consiste justamente en plantear cuál es el papel de la dirección escolar desde una perspectiva de la participación, la autonomía y la rendición de cuentas como nuevos elementos de "gobernanza" (Bolívar, 2012; Chapman, Lindsay, Muijs, Harris, Arweck, \& Goodall, 2010). Una forma de llevar los centros es aquella que apunta a modos más consensuados, cooperativos y horizontales, lo cual requiere estructuras y procesos de relación que favorezcan la corresponsabilidad de los profesores, apoyados por la dirección de la escuela.

De acuerdo a otros estudios realizados en el ámbito del ISSPP, los aspectos que caracterizan a los líderes exitosos es que éstos muestren a) una dirección con una orientación clara del rumbo que debe tomar la escuela, b) un liderazgo humano, centrado en el desarrollo integral de las personas, c) una promoción de la escuela como comunidad, d) una adecuación al contexto en el que se encuentra emplazada y e) un liderazgo pedagógico, preocupándose por que la metodología docente y el currículum sean innovadores y promuevan el cambio educativo (Leithwood, \& Riehl, 2005; Moos, 2009).

La dirección, en otro sentido, y la toma de decisiones de las organizaciones implica en el contexto español la comprensión de ésta de un modo democrático. En ella se abre la mirada a otros tipos de relación en las escuelas. Se analiza el centro educativo desde una doble visión: como Comunidad de Aprendizaje y como una Red de escuelas que se apoyan, siendo instituciones que aprenden. En este sentido, no deben estar sustentadas en una única persona, sino que los objetivos sean compartidos. Es una tarea más de equipo, un liderazgo distribuido, que busque el aprendizaje de los estudiantes desde una mirada transformadora de la sociedad que aspire a una mayor Justicia Social (Bolívar, Murillo y López-Yáñez, 2013).

Un aspecto crucial es la formación de los nuevos /as directores/as de los centros escolares. Este elemento ha sido destacado también en estudios como los de Chap- 
man, et al. (2010), Darling-Hammond, LaPointe, Meyerson, \& Orr (2007) y Storey (2016). En sus planes, al menos en el contexto español, se incide especialmente en los aspectos de gestión, y quizá su foco es mucho menor en nuevos modos de liderazgo o de gobernanza, a los que se tiende o se pretende: una dirección menos homogénea, menos burocrática. Sin embargo, este es el reto. Formar gente capaz de concitar una meta compartida por docentes y familias. Nos preguntamos si es posible formar, y más aún ejercer la dirección eficaz sin que se siga la lógica no burocrática que construya a los centros de forma que puedan seguir procesos de aprendizaje (Murillo y Hernández-Castilla, 2015; Murillo, 2016).

Para ello es necesario que los directores/directoras consideren el desarrollo de las personas como parte esencial de su tarea. Hay que tener en cuenta que la dirección ha de estar enfocada a la mejora de la escuela. En este sentido, es esencial una gestión más autónoma dirigida al aprendizaje de todos y cada uno de los estudiantes. El dilema es si a esta autonomía de los centros, de su gestión, de su dirección va acompañada de una mayor presión en el rendimiento de cuentas por parte de los centros (Vázquez Toledo, Liesa \& Bernal Agudo, 2016).

La orientación debe apuntar a una regulación menos rígida donde la confianza y la autonomía de los centros permita otros tipos de dirección. Seguramente hay que pensar nuevos modos de acceso, selección y formación de los directores y directoras de las escuelas. Es una tarea compartida y comunitaria. Esta tarea compartida está presente en los resultados también de esta investigación cuando se aborda el liderazgo de la comunidad (López-Yáñez, \& Lavié, 2010).

La principal fortaleza del presente estudio es que aporta datos relevantes sobre la realidad del liderazgo en España dentro del marco del ISSPP, proyecto de gran envergadura que respalda y apoya con datos internacionales los hallazgos de esta investigación. Por otro lado, los puntos débiles del estudio es que sería interesante analizar los datos de los distintos estudios del ISSPP realizados en España de forma complementaria, pudiendo tener así una imagen más completa y detallada del liderazgo exitoso español y compararlo con los resultados encontrados en estudios de otros países conformantes de la red ISSPP.

En el estudio de estos directores y directoras con un perfil de liderazgo escolar exitoso podemos afirmar que son docentes implicados en su escuela, entusiasmados por la docencia en el aula. Han sido votados por los representantes del claustro en el consejo escolar, los estudiantes y las familias mediante un proceso electoral. Pero además cuentan tanto con el reconocimiento de la comunidad educativa como del entorno donde están situados los centros. Estos líderes se caracterizan por promover una escuela más participativa, más integrada en la comunidad, más socialmente comprometida que activamente quiera no solo una escuela justa sino una sociedad de mayor Justicia Social (Murillo, \& Hernández-Castilla, 2011).

Como síntesis de los relevantes aportes del ISSPP al estudio del liderazgo, el liderazgo exitoso se puede sintetizar en cuatro elementos básicos: a) contribuye a la mejora del aprendizaje de los estudiantes, b) ha de estar distribuido entre otros miembros de la escuela y también la comunidad educativa, c) establece metas conjuntas y favorecen el desarrollo de las personas así como la redefinición de la 
organización y la gestión del currículum y d) promueve prácticas para atender la diversidad de los estudiantes y mejorar la calidad, la equidad y la Justicia Social.

\section{Referencias}

Álvarez, M. (2010). Liderazgo compartido: Buenas prácticas de dirección escolar. Madrid: Wolters Kluwer.

Bell. L., Bolam, R., \& Cubillo, L. (2003). A systematic review of the impact of school leadership and management on student outcomes. Londres: Cassell

Bennis, W. , \& Nanus, B. (2008). Líderes: Estrategias para un liderazgo eficaz. Barcelona: Paidos.

Bolivar, A. (2000). Los centros escolares como comunidades. Revisando la colegialidad. Revista Española de Pedagogía, 58(216) 253-274.

Bolívar, A. (2012). Melhorar os processos e os resultados educativos. O que nos ensina a investigação. Vila Nova de Gaia: Fundação Manuel Leão

Bolívar, A., López Yáñez, J. , \& Murillo, F. J. (2013). Liderazgo en las instituciones educativas. Una revisión de líneas de investigación. Revista Fuentes, 14, 15-60.

Crow, G; Day, C., \& Møller, J. (2016). Framing Research on School Principals' Identities. International Journal of Leadership in Education, 1, 1-13. doi: 10.1080/13603124.2015.1123299

Chapman, C., Lindsay, G., Muijs, D., Harris, A., Arweck, E. , \& Goodall, J. (2010). Governance, leadership, and management in federations of schools. School effectiveness and school improvement, 21(1), 53-74.

Darling-Hammond, L., LaPointe, M., Meyerson, D. , \& Orr, M. T. (2007). Preparing School Leaders for a Changing World: Lessons from Exemplary Leadership Development Programs. School Leadership Study. Stanford, CA: Stanford University. Recuperado de: www.wallacefoundation.org/knowledge-center/.../Preparing-School-Leaders.pdf

Day, C. (2017). Teachers' Worlds and Work: understanding complexity, building quality. Londres: Routledge.

Day, C, Gu, Q., \& Sammons, P. (2016) The Impact of Leadership on Student Outcomes: How Successful School Leaders Use Transformational and Instructional Strategies to Make a Difference. Educational Administration Quarterly, 52(2), 221-258. doi:10.1177/0013161X15616863

Day, C. (2016). Teachers and the quality of education: issues in capability, quality and teacher professional development. En M. Mulder (Ed.), Competence-based Vocational and Professional Education (pp. 165-182). Londres: Springer.

Day, C., \& Gurr, D. (2013). Leading Schools Successfully: Stories from the Field. Londres: Routledge.

Day, C., \& Leithwood, K. (2007). Successful principal leadership in times of change: An international perspective. Dordrecht: Springer.

Day, C., Sammons, P., Hopkins, D., Harris, A., Leithwood, K., Gu, Q., Brown, E., Ahtaridou, E. , \& Kington, A. (2009). The impact of school leadership on pupil outcomes. Nottingham: NCSL.

Day, C., Sammons, P., Leithwood, K., Hopkins, D., Harris, A., Gu, Q. , \& Brown, E. (2010). Ten strong claims about successful school leadership. Nottingham: The National College for School Leadership. 
Drysdale, L., \& Gurr, D. (2011). Theory and practice of successful school leadership in Australia. School Leadership \& Management, 31(4), 355-368. doi:10.1080/13632434.2011.606273

Drysdale, L. , \& Gurr, D. (2016). Reflections on successful school leadership from the International Successful School Principalship Project. En G. Lakomski, S. Eacott y C. W. Evers (Eds.), Questioning Leadership: New directions for educational organisations (pp. 187-204). Londres: Routledge.

Foster, W. (1986). The Reconstruction of Leadership. Victoria: Deakin University Press.

Fullan, M. (2003). The moral imperative of school leadership. Nueva York, NY: Corwin Press.

Gurr, D., \& Drysdale, L. (2003). Successful School Leadership: Victorian case studies. International Journal of Learning, 10, 945-957.

Gurr, D., Drysdale, L., \& Mulford, B. (2006). Models of successful principal leadership. School Leadership and Management, 26(4), 371-395. doi: 10.1080/13632430600886921

Hallinger, P., \& Heck, R. H. (1996). Reassessing the principal's role in school effectiveness: A review of empirical research: 1980-1995. Educational Administration Quarterly, 32(1), 5-44. doi: 10.1177/0013161X96032001002

Hargreaves, A., \& Fink, D. (2003). Sustaining leadership. En B. Davies y J. WestBurnham (Eds.), Handbook of Educational leadership and management (pp. 431-451). Londres: Pearson.

Hargreaves, A., \& Fink, D. (2008). Distributed leadership: Democracy or delivery?. Journal of Educational Administration, 46(2), 229-240.

Harris, A., Leithwood, K., Day, C., Sammons, P. , \& Hopkins, D. (2007). Distributed leadership and organizational change: Reviewing the evidence. Journal of Educational Change, 8(4), 337-347. doi:10.1007/s10833-007-9048-4

Hopkins, D., Ainscow, M., \& West, M. (1994). School Improvement in an Era of Change. Londres: EPPI.

Leithwood, K. (2005). Educational Leadership. A Review of the Research. Toronto: The Mid-Atlantic Regional Educational Laboratory.

Leithwood, K., \& Jantzi, D. (2006). Transformational school leadership for largescale reform: Effects on students, teachers, and their classroom practices. School Effectiveness and School Improvement, 17(2), 201-227. doi: 10.1080/09243450600565829

Leithwood, K., \& Riehl, C. (2005). What do we already know about educational leadership. En W. Firestone y C. Riehl (Eda.), A new agenda for research in educational leadership (pp. 12-27). Nueva York: Teachers College Press.

Leithwood, K., Seashore L. K., Anderson, S., \& Wahlstrom, K. (2004). Review of Research: How Leadership Influences Student Learning. Recuperado de http://www.wallacefoundation.org/knowledge-center/Pages/How-Leadership-Influences-StudentLearning.aspx

López-Yáñez, J., \& Lavié, J.M. (2010). Liderazgo para sostener procesos de innovación. Profesorado: Revista de Currículum y Formación del Profesorado, 14(1), 71-92.

Møller, J. (2016). Norway: Researching Norwegian Principals. En H. Arlestig, C. Day, y O. Johansson (Eds.), A Decade of Research on School Principals (pp. 77-101). Londres: Springer. 
Moos, L. (2009). Hard and soft governance: The journey from transnational agencies to school leadership. European Educational Research Journal, 8(3), 397-406. doi:10.2304/ eerj.2009.8.3.397

Moos, L., Johansson, O., \& Day, C. (Eds.). (2011). How school principals sustain success over time: International perspectives. Londres: Springer.

Mulford, W., Silins, H., \& Leithwood, K. A. (2004). Educational leadership for organisational learning and improved student outcomes. Londres: Springer.

Muñoz-Repiso, M. (1995). Calidad de la educación y eficacia de la escuela: estudio sobre la gestión de los recursos educativos. Madrid: Ministerio de Educación.

Murillo, F. J., \& Hernández-Castilla, R. (2015). Liderazgo para el aprendizaje:¿ Qué tareas de los directores y directoras escolares son las que más inciden en el aprendizaje de los estudiantes. RELIEVE, 21(1), art 1. doi: 10.7203/relieve.21.1.5015

Murillo, F. J. (2016). La investigación sobre liderazgo educativo: una mirada desde el presente proyectada al futuro. Revista Fuentes, 14, 9-14.

Robinson, V. (2007). The impact of leadership on student outcomes: Making sense of the evidence. Recuperado de http://research.acer.edu.au/research_conference_2007/5

Robinson, V. M., Lloyd, C. A., \& Rowe, K. J. (2008). The impact of leadership on student outcomes: An analysis of the differential effects of leadership types. Educational Administration Quarterly, 44(5), 635-674. doi: 10.1177/0013161X08321509

Silins, H., \& Mulford, B. (2004). Schools as learning organisations-Effects on teacher leadership and student outcomes. School Effectiveness and School Improvement, 15(34), 443-466. doi: 10.1080/09243450512331383272

Spillane, J. P., Camburn, E. M., \& Stitziel, A. (2007). Taking a distributed perspective to the school principal's workday. Leadership and Policy in Schools, 6(1), 103-125. doi:10.1080/15700760601091200

Spillane, J. P., Diamond, J. B., Burch, P., Hallett, T., Jita, L., \& Zoltners, J. (2002). Managing in the middle: School leaders and the enactment of accountability policy. Educational Policy, 16(5), 731-762. doi:10.1177/089590402237311

Stoll, L. , \& Fink, D. (1998). The cruising school: The unidentified ineffective school. En L. Stoll (Ed.), No quick fixes: Perspectives on schools in difficulty (pp. 189-206). Londres: Falmer Press.

Storey, J. (2016). Leadership in Organizations: Current Issues and Key Trends. Nueva York: Routledge.

Swann, R., Gurr, D., Ford, P., Drysdale, L., Hardy, R. y Di Natale, E. (2003). Successful school leadership in Victoria: three case studies. Leading and Managing, 9(1), 18-55.

Thyssen, O. (2003). Organisationens usynlighed. Distinktion: Scandinavian Journal of Social Theory, 4(2), 65-74.

Vázquez Toledo, S., Liesa Orús, M., \& Bernal Agudo , J.L. (2016). El camino hacia la profesionalización de la función directiva: el perfil competencial y la formación del director de centros educativos en España. Perfiles educativos, 38(151), 158-174.

Wang, L. H., Gurr, D. , \& Drysdale, L. (2016). Successful school leadership: case studies of four Singapore primary schools. Journal of Educational Administration, 54(3), 270-287. doi:10.1108/JEA-03-2015-0022 
Weick, K. E. (2001). Leadership as the legitimation of doubt. En W. Bennis, G. M. Spreitzer y T. G. Cummings (Eds.), The Future of Leadership: Today's Top Leadership Thinkers Speak to Tomorrow's Leaders (pp. 91-102). San Francisco: Jossey-Bass.

Fecha de recepción: 03 de enero de 2017

Fecha de revisión: 03 de enero de 2017

Fecha de aceptación: 28 de marzo de 2017 